\title{
Qualidade de vida de pacientes idosos submetidos à cirurgia de revascularização
}

\author{
Quality of life analysis in elderly \\ patients undergoing surgery for \\ coronary artery bypass grafting
}

FisiSenectus . Unochapecó Ano 2, n. 1 - Jan/Jun. 2014 p. $13-23$

Amanda Christina Monteiro Osório. amandasc@unochapeco.edu.br

Estudante do Curso de Graduação em Fisioterapia, Universidade Comunitária da Região de Chapecó (Unochapecó), Chapecó, SC, Brasil.

Liziane Flores. liziflores@unochapeco.edu.br

Estudante do Curso de Graduação em Fisioterapia, Universidade Comunitária da Região de Chapecó (Unochapecó), Chapecó, SC, Brasil.

Lilian Marin. fisiolili@unochapeco.edu.br Docente do Curso de Graduação em Fisioterapia, Universidade Comunitária da Região de Chapecó (Unochapecó), Chapecó, SC, Brasi.

\section{Sheila Cristina Cecagno Zanini.}

Mestranda em Envelhecimento Humano-PPGEH-UPF.

Camila Pereira Leguisamo. camila@upf.br

Docente do Programa de Mestrado em Envelhecimento Humano de Passo Fundo-UPFDoutora em Ciências da Saúde: Cardiologia, pelo Instituto de Cardiologia / Fundação Universitária de Cardiologia, Brasil.

\section{Resumo}

Introdução: A cirurgia de revascularização do miocárdio tem o intuito de melhorar a qualidade de vida relacionada à saúde, além de promover o alívio dos sintomas relacionados à cardiopatia e ainda aumentar a sobrevida. Objetivo: Neste estudo, objetivou-se avaliar a qualidade de vida no pré e pós-operatório de cardiopatas submetidos à cirurgia de revascularização do miocárdio, relacionando-a com a idade, a ansiedade e a dor. Metodologia: Trata-se de uma pesquisa de natureza quantitativa, observacional e transversal descritiva em que utilizou-se o questionário MacNew QLMI para avaliar a qualidade de vida, o Inventário de Ansiedade Traço-Estado (IDATE) para avaliar a ansiedade e a Escala Visual Analógica (EVA) para avaliar a dor. Resultados: Dentre os seis sujeitos avaliados, $90 \%$ eram homens, com média de idade de $70,5( \pm 8,43)$ anos. Dos fatores de risco, o mais presente foi a hipertensão arterial sistêmica (100\%), seguido do sedentarismo (83\%), dislipidemia (50\%) e tabagismo (50\%). Conclusão: Concluiu-se que a cirurgia de revascularização do miocárdio melhorou a qualidade de vida dos pacientes avaliados e estes mantiveram um nível médio da ansiedade entre o período pré e o pós-operatório. No que se refere à dor houve melhora comparando o pós ( $\left.15^{\circ} \mathrm{dia}\right)$ com o pré-operatório.

\section{Palavras-chave}

doença das coronárias, dor, qualidade de vida, cirurgia torácica, Fisi enectUS
fisioterapia. 


\begin{abstract}
Introduction: Coronary artery bypass grafting aims to improve the quality of life related to health, promote the relief of symptoms related to heart disease and also improve survival. Objective: This study aimed to evaluate the quality of life in pre-and post-operative cardiac patients undergoing coronary artery bypass grafting, relating to age, anxiety and pain. Materials and Methods: This is a quantitative research, observational and cross-sectional descriptive which used MacNew QLMI questionnaire to assess quality of life, the Trait Anxiety Inventory-State (STAI) to assess anxiety and Visual Analogue Scale (VAS) to assess pain. Results: Among the 06 subjects evaluated, $90 \%$ were men, with an average age of $70.5( \pm 8.43)$ years. From the risk factors, the most relevat was hypertension (100\%), followed by physical inactivity ( $83 \%$ ), dyslipidemia $(50 \%)$ and smoking (50\%). Conclusion: It was concluded that CABG surgery improved the quality of life of the patients and they remained a medium level of anxiety between the pre and post. As regards the pain had improved comparing the post (day 15) with the pre.
\end{abstract}

\title{
Keywords
}

coronary disease, pain, quality of life, thoracic surgery, physical therapy specialty.

\section{Introdução}

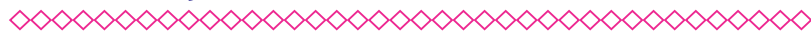

Atualmente, as doenças cardiovasculares têm sido consideradas um desafio para a medicina. 0 crescente número de pacientes com doenças do coração e o considerável aumento de óbitos vêm sendo amplamente discutidos na área da saúde, especialmente nos países em desenvolvimento e subdesenvolvidos ${ }^{1}$.

No Brasil, em 2020, essas doenças estarão entre as principais causas de morbimortalidade, contribuindo com 20 milhões de óbitos anualmente e aumentando para mais de 24 milhões em $2030^{2}$. Vários estudos têm evidenciado que apesar da expectativa de vida do brasileiro ter crescido nos últimos dez anos, em contrapartida houve um índice muito maior de doenças cardiovasculares ${ }^{3}$.

Contudo, o aumento na expectativa de vida, as melhorias das condições básicas de saúde, bem como o admirável avanço da medicina nos últimos anos, ainda fazem com que a cardiologia se depare com pacientes acima de 70 anos, sem que a idade seja entendida como um impedimento para a indicação de qualquer tipo de tratamento, resultando em um maior número de idosos submetidos à cirurgia cardíaca ${ }^{4}$.

No ano de 2011, no Brasil, foram realizadas aproximadamente 100 mil cirurgias cardíacas, estas objetivaram o regresso do bem-estar físico, mental e social do paciente e mais de $50 \%$ dessas cirurgias eram de revascularização miocárdica (RM), um dos procedimentos mais comuns no tratamento das doenças cardíacas, cujo indicador é comparável à literatura internacional 5,6.

As Diretrizes da Cirurgia de Revascularização Miocárdica, Valvopatias e Doenças da Aorta relatam que a revascularização miocárdica é uma das cirurgias mais realizadas em todo o mundo, considerando que, nas últimas três décadas, desde a realização da primeira revascularização direta do miocárdio, houveram muitos avanços, ligados, em suma, à revisão de vários conceitos concernentes à aterosclerose, tecnologia e técnica cirúrgica ${ }^{7}$.

Canguilhem ${ }^{8}$ mostra que a saúde envolve a capacidade de adoecer e sair do estado patológico, ou seja, a saúde é tida como a possibilidade de encarar situações novas, estando diretamente relacionada ao limiar de tolerância ou de segurança de cada indivíduo para enfrentar e superar as condições contrárias do meio. Dessa forma, a qualidade de vida passa a ter um importante papel na manutenção de uma condição favorável a saúde.

Franzen ${ }^{9}$ relata que o termo qualidade de vida engloba um conjunto de características, ditas como domínios, que incluem o estado físico, funcional, mental e social do indivíduo, resultando em um conceito subjetivo, multidimensional, que inclui elementos de avaliação tanto positivos quanto negativos. 0 avanço tecnológico juntamente 
com a medicina têm proporcionado à população maior segurança nas operações cardiovasculares de alta complexidade, no entanto, não só a tecnologia e a medicina são suficientes para oferecer ao paciente um programa cirúrgico cardiovascular de excelência, mas também uma equipe multiprofissional integrada ${ }^{10}$.

Considerando as modificações geradas pelo procedimento cirúrgico, uma das preocupações dos profissionais da área da saúde, mais especificamente os que estão envolvidos diretamente com o paciente, é de proporcionar que este tenha uma melhora da qualidade de vida, bem como que sua independência funcional seja garantida, permitindo o seu retorno às atividades da vida diária.

No Brasil, pesquisas que dão ênfase às doenças cardiovasculares, bem como às cirurgias cardíacas, relacionadas à avaliação qualidade de vida, dor e ansiedade, estão se tornando cada vez mais frequentes. Na região Sul os estudos são escassos, bem como no estado de Santa Catarina e na região Oeste, em que não há trabalhos dessa dimensão perfazendo-se a necessidade do desenvolvimento da pesquisa em questão.

Diante do exposto, a avaliação da qualidade de vida em saúde no pré e pós-operatório de pacientes cardiopatas é de extrema importância, visto que possibilita identificar componentes que podem ser alterados e que interferem diretamente na percepção individual. Sendo assim, o objetivo dessa pesquisa foi o de analisar a qualidade de vida de cardiopatas no pré e pós-operatório do procedimento cirúrgico de revascularização do miocárdio e verificar se houve melhora desta.

\section{Materiais e métodos}

$\infty \times \infty \times \infty \times \infty \times \infty \times \infty \times \infty \times \infty \times \infty \times \infty \times \infty \times \infty \times \infty$

A pesquisa realizada foi de natureza quantitativa, observacional e transversal descritiva. A população constituiu-se de 21 cardiopatas submetidos à cirurgia de revascularização do miocárdio no modo eletivo, no Hospital Regional São Paulo - Assec, no município de Xanxerê (SC), no período de abril a junho de 2013, contudo a amostra restringiu-se a seis indivíduos, maiores de 50 anos, de ambos os gêneros, inclusos conforme os critérios estabelecidos e que aceitaram participar da pes- quisa. Os demais foram excluídos por apresentarem instabilidade hemodinâmica, complicação neurológica grave (qualquer síndrome ou paralisia que afetem o cognitivo do paciente, impedindo-o de realizar as avaliações), confusão mental ou inconsciência, diabetes mellitus descompensada, obesidade mórbida, doenças degenerativas (câncer, mal de Alzheimer, esclerose múltipla, distrofias musculares e doença de Parkinson) e insuficiência renal aguda.

Primeiramente, foi realizado o contato com a instituição hospitalar a fim de obter a autorização por meio do termo de concordância entre as instituições. Depois que a pesquisa foi autorizada e aprovada pelo Comitê de Ética em Pesquisa da Universidade Comunitária da Região de Chapecó (Unochapecó), sob o protocolo nº. 340/12, os médicos cirurgiões cardiovasculares responsáveis pelas cirurgias de revascularização do miocárdio da unidade hospitalar envolvida foram contatados, para que indicassem os pacientes que participaram do estudo.

A coleta teve duração aproximada de 50 dias não contínuos, entre os meses de abril a junho de 2013, e foram utilizados os seguintes instrumentos de pesquisa: ficha de coleta de dados sociodemógraficos e clínicos adaptada, composta por itens que se referem aos dados pessoais, fatores predisponentes para a doença cardiovascular, informações sobre o procedimento cirúrgico e hospitalização ${ }^{11}$; Questionário MacNew Quality of Life after Myocardial Infarction Questionnaire (MacNew QLMI) para avaliar a percepção da qualidade de vida, composto de 27 itens que se dividem em três domínios: físico-funcional, emocional e social ${ }^{12}$; Inventário de Ansiedade Traço-Estado IDATE $^{13}$, traduzido e adaptado por Biaggio e Natalício ${ }^{14}$, composto de duas escalas, cada uma com 20 frases, sob a forma de escala Likert, que avaliam como o sujeito se sente no momento e a de como ele geralmente se sente ${ }^{14}$ e a Escala Visual Analógica (EVA), que proporciona uma medição simples e eficiente da intensidade da dor, assinalando uma linha horizontal com dez centímetros de comprimento, sendo que uma das extremidades corresponde ao termo "sem dor" e outra ao termo "dor máxima". As pontuações menores de 3,4 indicam "dor leve", pontuações entre 3,5 e 6,7 indicam "dor moderada" e maiores que 6,7 indicam "dor grave". 
Tal escala tem sido utilizada largamente na clínica e em laboratórios de investigação, na obtenção de índices rápidos da dor, possibilitando sua avaliação por meio de um valor numérico ${ }^{15,16}$.

Na primeira abordagem ao paciente, o termo de consentimento livre e esclarecido foi apresentado em duas vias, a fim de obter autorização formal do indivíduo para a coleta de dados, possibilitando- o a escolha de participar e, no caso de sentir-se constrangido, o direito de retirar-se da pesquisa. Ainda neste primeiro momento, após a assinatura do termo, os pacientes realizaram a primeira parte da pesquisa, respondendo aos questionários referentes ao pré-operatório.

Os questionários foram nominados através de algarismos romanos, garantindo sigilo absoluto, visto que os resultados são apresentados em valores numéricos, não possibilitando a identificação dos participantes. Foram aplicados de forma individual, tendo duração média de 30 minutos. Cada um foi aplicado três vezes, a primeira abordagem entre o sétimo e o terceiro dia de pré-operatório, e ainda no quinto e $15^{\circ}$ dia de pós-operatório, realizados na forma presencial e por contato telefônico (15ㅇia).

Os procedimentos estatísticos foram realizados utilizando-se SPSS Statistics 14.0. As variáveis categóricas são expressas como frequência absoluta, relativa e percentil, e as numéricas como média \pm desvio padrão.

Esta pesquisa seguiu as orientações estabelecidas pelo Conselho Nacional de Saúde para pesquisas com seres humanos e da Comissão Nacional de Ética em Pesquisa (Conep) por meio da Resolução n 196, de 10 de outubro de 1996, do Conselho Nacional de Saúde (BRASIL, 1996) e da Resolução nº 010 de 22 de setembro de 1978, do Conselho Federal de Fisioterapia e Terapia Ocupacional (Coffito) (BRASIL, 1978).

\section{Resultados}

$\infty \times \infty \times \infty \times \infty \times \infty \times \infty \times \infty \times \infty \times \infty \times \infty \times \infty \times \infty \times$

Depois de os seis indivíduos serem avaliados verificou-se, no que se refere ao gênero e à idade, que cinco foram do gênero masculino e um do feminino, com média de idade de $70,5( \pm 8,43)$ anos para ambos os gêneros. Quanto à cor e ao estado civil, $100 \%$ dos indivíduos eram de cor branca e casados. Quanto à escolaridade, observou-se que $33 \%$ dos indivíduos completaram o nível fundamental, $50 \%$ o nível médio e $17 \%$ o nível superior. No que se refere à atividade laboral, 33\% dos indivíduos eram aposentados, $50 \%$ eram agricultores e $17 \%$ eram representantes comerciais.

No que se refere aos fatores de risco para a doença cardiovascular, a Tabela 1 mostra que 100\% dos pacientes apresentaram hipertensão arterial sistêmica (HAS), sendo que apenas 17\% apresentaram diabetes mellitus (DM) associada, 50\% apresentaram dislipidemia, 17\% apresentaram depressão/ansiedade. Destes, $50 \%$ eram tabagistas há pelo menos dez anos e 83\% eram sedentários. Percebeu-se que os indivíduos apresentaram pelo menos dois fatores de risco de forma associada.

A Tabela 2 apresenta a prevalência de fatores associados à doença cardiovascular e à recuperação do paciente, em que: apenas 17\% apresentaram angioplastia prévia, 100\% apresentaram cateterismo prévio, nenhum realizou fisioterapia no período pré-operatório e $100 \%$ realizaram fisioterapia após a realização do procedimento cirúrgico durante a permanência no hospital. A permanência no âmbito hospitalar teve um tempo médio de $13( \pm 13,14)$ dias, entre os períodos pré e pós-operatório.

No que se refere à percepção da qualidade de vida, a categorização foi realizada com base no estudo de Matos (1999) ${ }^{17}$, no qual utilizou o mesmo instrumento para avaliar a qualidade de vida (QV). No referido estudo, a QV varia entre 1 e 7 pontos e é agrupada nos seguintes intervalos: 1 a 3, 3 a 5 e 5 a 7 . A média situada no intervalo [1 a 3] corresponde a uma pior QV, considera-se moderada QV a média situada entre os [3 e 5], e uma elevada QV em que a média situa-se entre os [5 e 7] pontos.

Conforme exposto na Tabela 3, os indivíduos apresentaram, no período pré-operatório, na dimensão emocional, uma média de 4,83 $( \pm 0,75)$ pontos, na dimensão física uma média de 4,50 $( \pm 1,22)$ pontos e na dimensão social uma média de $4,5( \pm 1,37)$ pontos, caracterizando, em todas as dimensões, uma moderada qualidade de vida.

Já no período pós-operatório, no quinto dia, na dimensão emocional houve uma média de 
$5,00( \pm 0,63)$ pontos, caracterizando uma elevada qualidade de vida, na dimensão física uma média de 4,33 $( \pm 0,81)$ pontos e na dimensão social uma média de $4,66( \pm 0,81)$ pontos, caracterizando uma moderada qualidade de vida.

Após 15 dias da realização do procedimento cirúrgico, a qualidade de vida na dimensão emocional apresentou uma média de $5,50( \pm 0,83)$ pontos, na dimensão física uma média de $5,00( \pm 0,63)$ pontos e na dimensão social uma média de 5,00 $( \pm 0,63)$ pontos, caracterizando uma elevada qualidade de vida.

Dessa forma, pôde-se observar que no período pré-operatório a qualidade de vida, considerando todas as dimensões, manteve-se moderada, meIhorando nos primeiros cinco dias de pós-operatório na dimensão emocional e após 15 dias caracterizando uma elevada qualidade de vida em todas as dimensões.

Quanto à ansiedade, a categorização mostra a ansiedade-estado como um estado emocional transitório ou condição do organismo humano caracterizado por sentimentos desagradáveis de tensão e apreensão conscientemente percebidos e por aumento na atividade do sistema nervoso autônomo. Já a ansiedade-traço refere-se às diferenças individuais relativamente estáveis em propensão à ansiedade, isto é, a diferença na tendência de reagir a situações percebidas como ameaçadoras com elevações na intensidade do estado de ansiedade. A pontuação foi realizada com base em estudo de Chaves (1994) ${ }^{18}$ em que de $20-40$ pontos há um baixo nível da ansiedade, de 40-60 pontos um nível médio da ansiedade e por fim, de 60-80 pontos um alto nível da ansiedade.

No período pré-operatório, no que se refere ao nível da ansiedade-estado, os indivíduos apresentaram média de $46,8( \pm 5,3)$ pontos e no nível da ansiedade-traço média de $45,3( \pm 6,6)$ pontos, caracterizando um nível médio da ansiedade em ambos e totalizando $92,1( \pm 9,49)$ pontos, conforme descrito na Tabela 4.

No pós-operatório tardio (quinto dia), no que se refere ao nível da ansiedade-estado, foi observada uma média de $41,16( \pm 7,27)$ pontos e no nível da ansiedade-traço média de $46,8( \pm 4,75)$ pontos, caracterizando um nível médio da ansiedade em ambos os casos e totalizando $88( \pm 9,35)$ pontos.
Já no pós-operatório ( $15^{\circ}$ dia) o nível da ansiedade-estado teve média de $42,16( \pm 5,98)$ pontos e o nível da ansiedade-traço média de 47,6 $( \pm 6,94)$ pontos, caracterizando um nível médio da ansiedade e totalizando $89,8( \pm 8,30)$ pontos.

Com os valores obtidos, percebeu-se que os indivíduos apresentaram um nível maior da ansiedade no pré-operatório, quando comparado ao pós-operatório tardio no quinto dia e com o $15^{\circ}$ dia, sendo que nestes últimos ocorreu, ainda, uma leve diminuição. Nesse sentido, pode-se observar, também, que a ansiedade, em todos os momentos em que foi avaliada, permaneceu-se em um nível médio.

A dor, avaliada no período pré-operatório, teve média de $3( \pm 3,52)$ na escala, caracterizando um quadro álgico leve. No pós-operatório tardio no quinto dia teve média de $5( \pm 0,89)$ na escala, caracterizando um quadro de dor moderado, já no pós-operatório referente ao $15^{\circ}$ dia teve média de $1( \pm 1,00)$ na escala correspondendo a uma dor leve, evidenciando a melhora do paciente após a alta hospitalar.

\section{Discussão}

$\infty<\infty<\infty<\infty<\infty<\infty<\infty<\infty<\infty<\infty<\infty<\infty<\infty<\infty<\infty<\infty<$

0 presente estudo demonstra que em uma população de cardiopatas submetidos à cirurgia de revascularização do miocárdio houve melhora na qualidade de vida quando comparado o pré-operatório com o pós-operatório, além da melhora no nível da ansiedade e da dor. Na literatura há poucos relatos com a utilização do instrumento MacNew QLMI para avaliação da qualidade de vida, fazendo-se necessária a incorporação deste à linguagem dos diversos ramos da ciência.

Os fatores de risco são os maiores predisponentes para doenças cardiovasculares e, consequentemente, para cirurgias cardíacas. Lima et al. ${ }^{19}$ realizaram estudo descritivo com o objetivo de verificar a frequência dos fatores de risco da doença coronariana em 78 pacientes que realizaram revascularização do miocárdio e encontraram a seguinte distribuição: hipertensão arterial $(83,3 \%)$, diabetes mellitus $(46,2 \%)$, dislipidemia $(46,2 \%)$, obesidade $(67,9 \%)$, sedentarismo $(57,7 \%)$ 
e tabagismo (34,6\%). Concluíram que a hipertensão arterial, a obesidade e o sedentarismo foram os fatores de risco mais frequentes nos pacientes que realizaram revascularização do miocárdio e que a maior parte deles apresentou no mínimo três fatores de risco.

Nesse mesmo sentido, o estudo de Fernandes, Aliti e Souza ${ }^{20}$ identificou o perfil clínico e cirúrgico de 58 pacientes submetidos à RM desde a sua chegada até a alta ou até o sétimo dia de permanência na unidade de terapia intensiva (UTI), observando a média de idade de 65,3 $( \pm 9,7)$ anos, a predominância do sexo masculino $(70,7 \%)$, sendo a hipertensão arterial sistêmica a comorbidade mais prevalente $(86,2 \%)$, seguida por dislipidemia $(60,3 \%)$ e diabetes mellitus (36,2\%). Na pesquisa, constatou-se que os pacientes submetidos à RM são majoritariamente homens, idosos, com fatores de risco clássicos para cardiopatia isquêmica.

Em estudo de Costa et al. ${ }^{21}$ que teve como objetivo traçar o perfil de pacientes submetidos à cirurgia de revascularização do miocárdio em que foram avaliados 48 indivíduos teve como resultados: a idade dos pacientes variou de 47 a 85 anos, com média de 62 anos, 33\% dos pacientes eram do sexo feminino e $67 \%$ do sexo masculino e quanto ao atendimento fisioterapêutico, foram prestados 763 terapias completando um total de 429 dias, 0 que sugere a média de dois atendimentos diários para cada paciente, sendo que o tempo de internação na UTI foi $3( \pm 1)$ dias e o tempo de internação na enfermaria foi de $6( \pm 3)$ dias, perfazendo um total de permanência hospitalar de $10( \pm 5)$ dias.

Alves Junior et al. ${ }^{22}$ realizaram uma pesquisa com 783 pacientes submetidos a cirurgias cardíacas valvares e de revascularização do miocárdio e evidenciaram que 197 pacientes tinham 70 anos ou mais, sendo que a média de idade foi de 74,1 $( \pm 3,9)$ e $61 \%$ eram do sexo masculino.

Gois, Dantas e Torrati23 utilizaram o SF-36 em um estudo descritivo e longitudinal objetivando comparar a qualidade de vida relacionada à saúde (QRVS) antes e seis meses após cirurgia de revascularização do miocárdio (CRM) e avaliar a sua relação com idade, sexo, escolaridade e estado civil. Entre os 54 sujeitos, 53,7\% eram homens, 66,7\% casados, com média de idade de $57,3( \pm 9,7)$ anos. Concluíram que não houve associação entre QVRS, escolaridade e estado civil e que a CRM melhorou a QVRS dos participantes.

Takiuti et al. ${ }^{24}$ utilizaram o Short-Form Health Survey (SF36) questionnaire em 483 pacientes com o objetivo de avaliar a qualidade de vida (QV) na doença multiarterial coronariana em pacientes submetidos randomicamente a cirurgia, angioplastia ou tratamento clínico. Destes, 161 foram revascularizados, 166 receberam angioplastia e 153 tiveram tratamento clínico. Observaram meIhora em todos os domínios e nas três opções terapêuticas. Comparativamente, a cirurgia ofereceu melhor qualidade de vida após quatro anos de seguimento.

Em estudo realizado por Gonçalves et al. ${ }^{25}$ utilizando o questionário MOS SF-36 o qual teve como objetivo avaliar a qualidade de vida de pacientes submetidos à cirurgia de revascularização do miocárdio em um programa de reabilitação cardíaca, foram observados 24 indivíduos de ambos os sexos ( 15 homens e 9 mulheres) na faixa etária entre 23 e 77 anos (média de idade de $58 \pm$ 6 anos) com quadro clínico estável. 0 questionário foi aplicado em três momentos: antes da cirurgia, no quinto dia do pós-operatório e dois meses após a cirurgia. Foi observada uma queda dos seguintes parâmetros: funcionamento do organismo, limitação por distúrbios físicos, vitalidade e dor. A conclusão do estudo foi que o questionário MOS SF-36 permite avaliar os benefícios da reabilitação cardíaca fase I a qual proporcionou autoconfiança e retorno às atividades diárias.

Em estudo prospectivo, utilizando o Inventário de Depressão de Beck (BDI), o Inventário de Ansiedade de Beck (BAI) e o teste de qualidade de vida (WHOQOL), 168 pacientes de ambos os sexos, entre 35 a 65 anos, foram observados a fim de investigar a frequência da depressão em indivíduos cardiopatas, com e sem diagnóstico de Infarto Agudo do Miocárdio (IAM), e em indivíduos sem diagnóstico de cardiopatia. Os resultados encontrados sugerem que os transtornos de depressão não são desencadeados pelo IAM, mas que estão presentes antes da admissão hospitalar, destacando a importância do rastreamento dos pacientes portadores de doença coronariana crônica ${ }^{26}$.

No estudo realizado por Saccomann, Cintra e Gallani27 foi avaliada a QV de 170 idosos com in- 
suficiência cardíaca em seguimento ambulatorial, utilizando o questionário de Minnesota. Verificou-se que houve menor influência da doença sobre a dimensão emocional, porém, com relação às questões vinculadas à dimensão física, houve maior impacto na qualidade de vida.

Garbossa et al. ${ }^{28}$ realizaram um ensaio clínico randomizado com 51 indivíduos com o intuito de verificar os efeitos de orientações fisioterapêuticas sobre o nível de ansiedade em pacientes submetidos à cirurgia de revascularização do miocárdio. Os pacientes foram avaliados através da Escala de Beck e da Escala Análogo Visual nos períodos pré e pós-operatório. Observaram escores de ansiedade mais baixos nos pacientes que receberam a intervenção antes da cirurgia e, além disso, percebeu-se que os indivíduos do gênero feminino apresentaram mais ansiedade no pré-operatório se comparados aos do gênero masculino. Observaram, ainda, a relação entre o tempo de permanência no hospital e ansiedade pós-operatória, sendo que os indivíduos mais ansiosos permaneceram internados por maior período de tempo. Dessa forma, concluíram que a orientação e instrução quanto a exercícios ventilatórios fisioterapêuticos e rotinas hospitalares, tiveram menores niveis de ansiedade no pré-operatório, entretanto, no período pós-operatório, ambos os grupos tiveram seus níveis de ansiedade reduzidos, sem diferença significativa entre eles.

Conforme pesquisa de Carneiro et al. ${ }^{29}$ os pacientes com doença cardíaca a serem submetidos a procedimentos invasivos e/ou cirúrgicos como o Estudo Eletrofisiológico (EEF), o implante de marcapasso e a revascularização do miocárdio apresentam prevalência de ansiedade e de depressão elevada e já os pacientes a serem submetidos ao EEF tiveram nível de ansiedade mais elevado que os outros. Concluíram, também, que a menor frequência de ansiedade foi observada precisamente no grupo de indivíduos submetidos à RM, o que não era esperado.

Com os dados analisados pode-se concluir que os indivíduos avaliados apresentaram Hipertensão Arterial Sistêmica (HAS) como principal fator de risco cardiovascular, seguido pelo sedentarismo, porém, não apresentaram níveis elevados de ansiedade e dor tanto no período pré-operatório quanto no pós-operatório da cirurgia de revascularização do miocárdio, o que não demonstrou alteração significativa no que se refere à qualidade de vida.

Tais resultados podem ser justificados pelo número restrito da amostra, pelo fato de a cirurgia ter sido realizada de modo eletivo, pela existência de poucos fatores de risco para doença cardiovascular e, ainda, devido a apenas um indivíduo ter realizado uma intervenção prévia (angioplastia). Esses fatores impedem uma interpretação acurada do comportamento da qualidade de vida, entretanto, essas informações podem ser úteis para elaboração, desenho e execução de estudos futuros na área com diferentes populações, bem como um número maior de amostra.

\section{Agradecimentos}

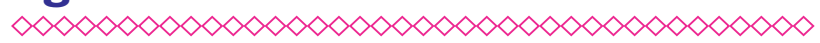

À equipe administrativa e médica do Hospital São Paulo de Xanxerê - SC pela pronta resposta nos contatos realizados, por nos permitir realizar este estudo e colaborar no fornecimento dos dados necessários, e aos pacientes que aceitaram participar desta pesquisa.

\section{Referências}

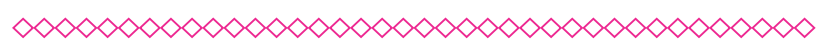

1. Christmann M, Costa CC, Moussalle LD. Avaliação da qualidade de vida de pacientes cardiopatas internados em um hospital público. Porto Alegre: Revista da AMRIGS. 2011; 55(3): 239-243.

2. Azambuja MIR, Foppa M, Maranhão MFC, Achutti AC. Impacto econômico dos casos de doença cardiovascular grave no Brasil: uma estimativa baseada em dados secundários. Arq Bras de Cardiol. 2008; 91(3): 163-171.

3. Dutra OP. II Diretriz brasileira de cardiopatia grave. Arq. Bras. Cardiol. 2006; 87(2): 223-232.

4. Milani R, Brofman P, Varela A, Souza JA, Guimarães M, Pantarolli R et al. Revascularização do miocárdio sem circulação extracorpórea em pacientes acima de 75 anos: análise dos 
resultados imediatos. Arq. Bras. Cardiol. 2005; 84(1):34-37.

5. Brail DM, Godoy MF. History of heart surgery in the world. Rev Bras Cir Cardiovasc. 2012; 27(1): 125-34

6. Morais DB, Lopes ACR, Sá VM de, Silva Júnior WM da, Cerqueira Neto ML de. Avaliação do desempenho funcional em pacientes submetidos à cirurgia cardíaca. Rev Bras Cardiol. 2010; 23(5): 263-269.

7. Brick AV, Souza DSR de, Braile DM, Buffolo $E$, Lucchese FA, Silva FP de V et al. Diretrizes da cirurgia de revascularização miocárdica, valvopatias e doenças da aorta. Arq. Bras. Cardiol. São Paulo, 2012.

8. Canguilhem, G. 0 normal e o patológico. 5. ed. Rio de Janeiro: Forense Universitária, 2002;307.

9. Franzen E. Avaliação de qualidade de vida em cardiopatia isquêmica: validação de instrumentos para uma população brasileira. Dissertação [mestrado]. Universidade Federal do Rio Grande do Sul. Faculdade de Medicina. Programa de Pósgraduação em Ciências Cardiovasculares. Porto Alegre, 2005; 107.

10. Atik FA, Garcia MFMA, Santos LM, Chaves $\mathrm{RB}$, Faber CN, Corso R B et al. Resultados da implementação de modelo organizacional de um serviço de cirurgia cardiovascular. Rev Bras Cir Cardiovasc. 2009; 24(2): 116-125.

11. Alcântara EC. Qualidade de vida após infarto agudo do miocárdio: avaliação com os questionários Mac New QLMI e SF-36. Dissertação [mestrado] Faculdade de Medicina. Universidade Federal de Uberlândia. Uberlândia-MG, 2005.

12. Benetti M, Nahas MV, Barros, MVG.

Reproducibility and validity of a brazilian version of the MacNew quality of life after myocardial infarction (MacNew QLMI) questionnaire. Med Sci Sports Exerc. 2001;33:62.

13. Spielberger CD, Gorsuch RL, Lushene, RD. STAI: manual for the State - Trait Anxiety Invetory. Palo Alto, CA: Consulting Psychologists Press, 1970.
14. Biaggio A, Natalicio LF. Manual para Inventário de Ansiedade Traço-Estado IDATE (Manual for Inventory Anxiety Trace-State-STAI), Rio de Janeiro: CEPA. 1979.

15. Sousa FAEF, Hortense P. Mensuração da dor. In: Chaves LD, Leão ER orgs. Dor: $5^{\circ}$ sinal vital: reflexões e intervenções da enfermagem. Curitiba: Ed. Maio. 2004; 7:75-84.

16. Bird SB, Dickson EW. Clinically significant changes in pain along the visual analog scale. Ann Emerg. Med. 2001; 38:639-43

17. Matos S. Qualidade de Vida dos Indivíduos após Enfarte Agudo doMiocárdio. Monografia realizada no âmbito do Curso de Estudos Superiores Especializados em Enfermagem Médico Cirúrgica, Escola Superior de Enfermagem Dr. Ângelo da Fonseca. Coimbra: 1999.

18. Chaves EC. Stress e trabalho do enfermeiro: a influência de características individuais no ajustamento e tolerância ao turno noturno. São Paulo: Instituto de Psicologia da Universidade de São Paulo, 1994.

19. Lima FET, Araújo TL, Lopes MVO, Silva LF, Monteiro ARM, Oliveira SKP. Fatores de risco da doença coronariana em pacientes que realizaram revascularização miocárdica. Rev Rene. 2012; 13(4): 853-60.

20. Fernandes MVB, Aliti G, Souza EN. Perfil de pacientes submetidos à cirurgia de revascularização miocárdica: implicações para o cuidado de enfermagem. Rev. Eletr. Enf. 2009; 11(4): 993-9.

21. Costa, CG et al. Perfil dos pacientes submetidos à Cirurgia de Revascularização do Miocárdio no Hospital de Cardiologia da Associação de Caridade Santa Casa do Rio Grande. Rio Grande: Sistema Anhanguera de Iniciação Científica; 2011.

22. Alves Júnior $L$, Rodrigues AJ, Évora PRB, Basseto S, Scorzoni Filho A, Luciano PM, Pinheiro KS, Vicente WVA. Fatores de risco em septuagenários ou mais idosos submetidos à revascularização do miocárdio e ou operações valvares. Rev Bras Cir Cardiovasc. 2008; 23(4): 550-555. 
23. Gois CFL, Dantas RAS, Torrati FG. Qualidade de vida relacionada à saúde antes e seis meses após a revascularização do miocárdio. Rev Gaúcha Enferm. Porto Alegre. 2009; 30(4):700-7.

24. Takiuti ME, Hueb W, Hiscock SB, Nogueira CRSR, Girardi P, Fernandes F et al . Qualidade de vida após revascularização cirúrgica do miocárdio, angioplastia ou tratamento clínico. Arq Bras Cardiol. 2007; 88(5):537-544.

25. Gonçalves FDP, Marinho PEM, Maciel MA, Galindo Filho VC, Dornelas AA de. Avaliação da qualidade de vida pós-cirurgia cardíaca na fase I da reabilitação através do questionário MOS SF36. Rev. Bras. Fisioter. 2006; 10(1):121-126.

26. Lemos C, Gottschall CAM, Pellanda LC, Muller M. Associação entre depressão, ansiedade e qualidade de vida após infarto do miocárdio. Psicologia: Teoria e Pesquisa. 2008; 24(4): 471476.

27. Saccomann ICR, Cintra FA, Gallani MCBJ. Qualidade de vida relacionada à saúde em idosos com insuficiência cardíaca: avaliação com instrumento específico. Acta Paul Enferm. 2011; 24(2):179-84.

28. Garbossa A, Maldaner E, Mortari DM, Biasi J, Leguisamo CP. Efeitos de orientações fisioterapêuticas sobre a ansiedade de pacientes submetidos à cirurgia de revascularização miocárdica. Rev Bras Cir Cardiovasc. 2009; 24(3): 359-366.

29. Carneiro AF, Mathias LAST, Júnior AR, De Morais NS, Gozzani JL, De Miranda AP. Avaliação da ansiedade e depressão no período pré-operatório em pacientes submetidos a procedimentos cardíacos invasivos. Rev Bras Anestesiol. 2009; 59(4): 431-438. 


\section{Anexos}

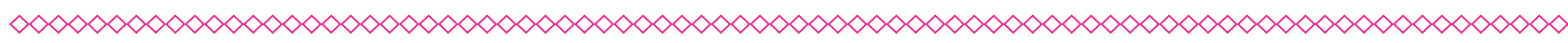

Tabela 1.

\begin{tabular}{|c|c|c|c|c|c|c|}
\hline \multicolumn{7}{|c|}{ Fatores de risco para doença cardiovascular } \\
\hline Paciente & HAS* & DM* & Dislipidemia & $\begin{array}{c}\text { Depressão/ } \\
\text { ansiedade }\end{array}$ & Tabagismo & Sedentarismo \\
\hline 01 & $\mathrm{X}$ & $\mathrm{X}$ & $\mathrm{X}$ & & & $\mathrm{X}$ \\
\hline 02 & $\mathrm{X}$ & & & & $\mathrm{x}$ & $\mathrm{x}$ \\
\hline 03 & $\mathrm{X}$ & & $\mathrm{X}$ & & & $\mathrm{X}$ \\
\hline 04 & $\mathrm{X}$ & & $\mathrm{X}$ & & $\mathrm{X}$ & $\mathrm{X}$ \\
\hline 05 & $\mathrm{X}$ & & & & $\mathrm{X}$ & $\mathrm{x}$ \\
\hline 06 & $\mathrm{X}$ & & & 01 & 03 & 05 \\
\hline TOTAL & 06 & 01 & 03 & 17 & 50 & 83 \\
\hline$\%$ & 100 & 17 & 50 & & & \\
\hline
\end{tabular}

Fonte: Dados da pesquisa

* HAS: Hipertensão Arterial Sistêmica, "DM: Diabetes mellitus

Tabela 2.

\begin{tabular}{|c|c|c|c|c|}
\hline \multicolumn{5}{|c|}{ Fatores associados } \\
\hline Paciente & $\begin{array}{c}\text { Angioplastia } \\
\text { prévia }\end{array}$ & $\begin{array}{c}\text { Cateterismo } \\
\text { prévio }\end{array}$ & $\begin{array}{c}\text { Fisioterapia } \\
\text { Pré }\end{array}$ & $\begin{array}{c}\text { Fisioterapia } \\
\text { Pós* }\end{array}$ \\
\hline 01 & & $X$ & & $X$ \\
\hline 02 & $\mathrm{X}$ & $\mathrm{X}$ & & $\mathrm{X}$ \\
\hline 03 & & $\mathrm{X}$ & & $\mathrm{X}$ \\
\hline 04 & & $\mathrm{X}$ & & $\mathrm{X}$ \\
\hline 05 & & $\mathrm{X}$ & & $\mathrm{X}$ \\
\hline 06 & 01 & 06 & 00 & 06 \\
\hline TOTAL & 17 & 100 & 00 & 100 \\
\hline$\%$ & & & & \\
\hline
\end{tabular}

Fonte: Dados da pesquisa.

* Duas vezes ao dia durante o período de internação. 
Tabela 3.

\begin{tabular}{|c|c|c|c|c|c|c|c|}
\hline \multicolumn{7}{|c|}{ Percepção da qualidade de vida } \\
\hline Paciente & $\mathbf{0 1}$ & $\mathbf{0 2}$ & $\mathbf{0 3}$ & $\mathbf{0 4}$ & $\mathbf{0 5}$ & $\mathbf{0 6}$ & Média $( \pm \mathrm{DP})^{*}$ \\
\hline Pré & & & & & & & \\
\hline $\begin{array}{c}\text { Emocional } \\
\text { Físico } \\
\text { Social }\end{array}$ & 4 & 4 & 5 & 5 & 6 & 4 & $4,83( \pm 0,75)$ \\
\cline { 2 - 9 } & 3 & 4 & 6 & 4 & 6 & 4 & $4,50( \pm 1,22)$ \\
\hline Pós (5 dias) & 3 & 4 & 6 & 3 & 6 & 5 & $4,50( \pm 1,37)$ \\
\hline $\begin{array}{c}\text { Emocional } \\
\text { Físico } \\
\text { Social }\end{array}$ & 5 & 5 & 5 & 5 & 6 & 4 & $5,00( \pm 0,63)$ \\
\cline { 2 - 9 } & 5 & 5 & 4 & 4 & 5 & 3 & $4,33( \pm 0,81)$ \\
\hline Pós (15 dias) & 5 & 5 & 4 & 4 & 6 & 4 & $4,66( \pm 0,81)$ \\
\hline Emocional & 6 & 6 & 6 & 5 & 6 & 4 & $5,50( \pm 0,83)$ \\
\hline $\begin{array}{c}\text { Físico } \\
\text { Social }\end{array}$ & 5 & 5 & 5 & 4 & 6 & 5 & $5,00( \pm 0,63)$ \\
\cline { 2 - 9 } & 5 & 5 & 5 & 4 & 6 & 5 & $5,00( \pm 0,63)$ \\
\hline
\end{tabular}

Fonte: Dados da pesquisa

Os dados foram avaliados através do Questionário MacNew Quality of Life after Myocardial Infarction Questionnaire (MacNew QLMI), (BENETTI; NAHAS; BARROS, 2001).

(clique para voltar ao texto) * $( \pm)$ desvio padrão

Tabela 4.

\begin{tabular}{|c|c|c|c|c|c|c|c|}
\hline \multicolumn{8}{|c|}{ Nível de ansiedade } \\
\hline Paciente & 01 & 02 & 03 & 04 & 05 & 06 & Média $( \pm \mathrm{DP}) *$ \\
\hline \multicolumn{8}{|l|}{ Pré } \\
\hline \multirow{3}{*}{$\begin{array}{c}\text { Traço } \\
\text { Estado } \\
\text { Total }\end{array}$} & 53 & 42 & 48 & 41 & 44 & 53 & $46,83( \pm 5,34)$ \\
\hline & 47 & 40 & 45 & 38 & 57 & 45 & $45,33( \pm 6,65)$ \\
\hline & 100 & 82 & 93 & 79 & 101 & 98 & $92,16( \pm 9,49)$ \\
\hline \multicolumn{8}{|l|}{ Pós (5 dias) } \\
\hline \multirow{3}{*}{$\begin{array}{c}\text { Traço } \\
\text { Estado } \\
\text { Total }\end{array}$} & 53 & 41 & 51 & 47 & 42 & 47 & $46,83( \pm 4,75)$ \\
\hline & 38 & 40 & 54 & 40 & 43 & 32 & $41,16( \pm 7,27)$ \\
\hline & 91 & 81 & 105 & 87 & 85 & 79 & $88,00( \pm 9,35)$ \\
\hline \multicolumn{8}{|l|}{ Pós (15 dias) } \\
\hline \multirow{3}{*}{$\begin{array}{c}\text { Traço } \\
\text { Estado } \\
\text { Total }\end{array}$} & 48 & 42 & 38 & 53 & 57 & 48 & $47,66( \pm 6,94)$ \\
\hline & 48 & 45 & 39 & 32 & 42 & 47 & $42,16( \pm 5,98)$ \\
\hline & 96 & 87 & 77 & 85 & 99 & 95 & $89,83( \pm 8,30)$ \\
\hline
\end{tabular}

Fonte: Dados da pesquisa

Os dados foram avaliados através do Inventário de Ansiedade Traço-Estado - IDATE (SPIELBERGER; GORSUCH; LUSHENE, 1970). 